\title{
Theory of optimal control using bisimulations
}

\author{
Mireille Broucke $^{\dagger}$, Maria Domenica Di Benedetto ${ }^{\ddagger \dagger}$, Stefano Di Gennaro ${ }^{\ddagger}$, and \\ Alberto Sangiovanni-Vincentelli ${ }^{\dagger}$ \\ $\dagger$ Dept. of Electrical Engineering and Computer Sciences, University of California at Berkeley, CA \\ 94720, USA. Tel: +11 510 642-1792; Fax: +11 510 643-5052; \\ ‡ Dip. di Ingegneria Elettrica, Università di L'Aquila, Poggio di Roio, 67040 L'Aquila, Italy. \\ mire, marika, alberto@eecs . berkeley . edu, digennar@dis . uniroma1.it
}

\begin{abstract}
We consider the synthesis of optimal controls for continuous feedback systems by recasting the problem to a hybrid optimal control problem: to synthesize optimal enabling conditions for switching between locations in which the control is constant. An algorithmic solution is obtained by translating the hybrid automaton to a finite automaton using a bisimulation and formulating a dynamic programming problem with extra conditions to ensure non-Zenoness of trajectories. We show that the discrete value function converges to the viscosity solution of the Hamilton-Jacobi-Bellman equation as a discretization parameter tends to zero.
\end{abstract}

\section{Introduction}

The goal of this paper is the development of a computationally appealing technique for synthesizing optimal controls for continuous feedback systems $\dot{x}=$ $f(x, u)$, by reducing substantially the complexity of the problem. This goal is achieved by virtue of recasting the problem to a hybrid optimal control problem. The hybrid problem is obtained by approximating the control set $U \subset \mathbb{R}^{m}$ by a finite set $\Sigma \subset U$ and defining vector fields for the locations of the hybrid system of the form $f(x, \sigma), \sigma \in \Sigma$; that is, the control is constant in each location. The hybrid control problem is, then, to synthesize an optimal switching rule between locations, or equivalently, optimal enabling conditions, such that a target set $\Omega_{f} \subset \Omega$ is reached while a hybrid cost function is minimized, for each initial condition in a specified set $\Omega \subset \mathbb{R}^{n}$.

Casting the problem into the domain of hybrid control is not appealing per se, on the contrary! Algorithmic approaches for solving the controller synthesis problem for specific classes of hybrid systems have appeared $[8,12]$ but no general, efficient algorithm is yet available. Hence, to be able to solve the (nonlinear) hybrid optimal control problem, we must exploit some additional property. We have a feasible and quite appealing approach if we can translate the problem to an equivalent discrete problem, which abstracts completely the continuous behavior. This translation is possible if we can construct a finite bisimulation defined on the hybrid state set. The bisimulation can be constructed using the geometric approach reported in [4], based on the following key assumption: $n-1$ local (on $\Omega$ ) first integrals can be expressed analytically for each vector field 
$f(x, \sigma), \sigma \in \Sigma$. This assumption is imposed in the transient phase of a feedback system's response, when the vector field is non-vanishing and local first integrals always exist, though analytical expressions for them may not be readily computable.

If the assumption is met, then we can transform the hybrid system to a finite automaton. The control problem posed on the finite automaton is to synthesize a discrete supervisor, providing a switching rule between automaton locations, that minimizes a discrete cost function approximating the original cost function, for each initial discrete state. We provide a dynamic programming solution to this problem, with extra constraints to ensure non-Zenoness of the closed-loop trajectories. By imposing non-Zeno conditions on the synthesis we obtain piecewise constant controls. The discrete value function depends on the discretizations of $U$ and of $\Omega$ using the bisimulation. We quantify these discretizations by parameters $\delta$ and $\delta_{Q}$, respectively. The main theoretical contribution is to show that as $\delta, \delta_{Q} \rightarrow 0$, the discrete value function converges to the unique viscosity solution of the Hamilton-Jacobi-Bellman (HJB) Equation.

There is a similarity between our approach to optimal control and regular synthesis, introduced in [2], in the sense that both restrict the class of controls to a set that has some desired property and both use a finite partition to define switching behavior. Our work provides a constructive approach to obtain the cell decomposition by using a finite bisimulation, which further allows us to formulate the synthesis problem on its quotient system - a finite automaton. The idea of using a time abstract model formed by partitioning the continuous state space has been pursued in a number of papers recently. Lemmon, Antsaklis, Stiver and coworkers [10] use a partition of the state space to convert a hybrid model to a discrete event system (DES). This enables them to apply controller synthesis for DES's to synthesize a supervisor. While our approach is related to this methodology, it differs in that we have explicit conditions for obtaining the partition. In [9] hybrid systems consisting of a linear time-invariant system and a discrete controller that has access to a quantized version of the linear system's output is considered. This approach suffers from spurious solutions that must be trimmed from the automaton behavior. Hybrid optimal control problems have been studied in papers by Witsenhausen [11] and Branicky, Borkar, Mitter [3]. These studies concentrate on problems of well-posedness, necessary conditions, and existence of optimal solutions but do not provide algorithmic solutions.

\section{Optimal control problem}

Notation. $\mathbf{1}(\cdot)$ is the indicator function. $\operatorname{cl}(A)$ denotes the closure of set $A$. $\|\cdot\|$ denotes the Euclidean norm. Let $C^{1}\left(\mathbb{R}^{n}\right)$ and $\mathcal{X}\left(\mathbb{R}^{n}\right)$ denote the sets of continuously differentiable real-valued functions and smooth vector fields on $\mathbb{R}^{n}$, respectively. $\phi_{t}\left(x_{0}, \mu\right)$ denotes the trajectory of $\dot{x}=f(x, \mu)$ starting from $x_{0}$ and using control $\mu(\cdot)$.

Let $U$ be a compact subset of $\mathbb{R}^{m}, \Omega$ an open, bounded, connected subset of $\mathbb{R}^{n}$, and $\Omega_{f}$ a compact subset of $\Omega$. Define $\mathcal{U}_{m}$ to be the set of measurable functions mapping $[0, T]$ to $U$. We define the minimum hitting time $T: \mathbb{R}^{n} \times$ $\mathcal{U}_{m} \rightarrow \mathbb{R}^{+}$by 


$$
T(x, \mu):= \begin{cases}\infty & \text { if }\left\{t \mid \phi_{t}(x, \mu) \in \Omega_{f}\right\}=\emptyset \\ \min \left\{t \mid \phi_{t}(x, \mu) \in \Omega_{f}\right\} & \text { otherwise. }\end{cases}
$$

A control $\mu \in \mathcal{U}_{m}$ specified on $[0, T]$ is admissible for $x \in \Omega$ if $\phi_{t}(x, \mu) \in \Omega$ for all $t \in[0, T]$. The set of admissible controls for $x$ is denoted $\mathcal{U}_{x}$. Let

$$
\mathcal{R}:=\left\{x \in \mathbb{R}^{n} \mid \exists \mu \in \mathcal{U}_{x} . T(x, \mu)<\infty\right\} .
$$

We consider the following optimal control problem. Given $y \in \Omega$,

$$
\begin{array}{rlr}
\text { minimize } & J(y, \mu)=\int_{0}^{T(y, \mu)} L(x(t), \mu(t)) d t+h(x(T(y, \mu))) \\
\text { subject to } & \dot{x}=f(x, \mu), \quad \text { a.e. } t \in[0, T(y, \mu)] \\
& x(0)=y
\end{array}
$$

among all admissible controls $\mu \in \mathcal{U}_{y} . J: \mathbb{R}^{n} \times \mathcal{U}_{m} \rightarrow \mathbb{R}$ is the cost-to-go function, $h: \mathbb{R}^{n} \rightarrow \mathbb{R}$ is the terminal cost, and $L: \mathbb{R}^{n} \times \mathbb{R}^{m} \rightarrow \mathbb{R}$ is the instantaneous cost. At $T(y, \mu)$ the terminal cost $h(x(T(y, \mu)))$ is incurred and the dynamics are stopped. The control objective is to reach $\Omega_{f}$ from $y \in \Omega$ with minimum cost.

Assumption 2.1.

(1) $f: \mathbb{R}^{n} \times \mathbb{R}^{m} \rightarrow \mathbb{R}^{n}$ satisfies $\left\|f\left(x^{\prime}, u^{\prime}\right)-f(x, u)\right\| \leq L_{f}\left[\left\|x^{\prime}-x\right\|+\left\|u^{\prime}-u\right\|\right]$ for some $L_{f}>0$. Let $M_{f}$ be the upper bound of $\|f(x, u)\|$ on $\Omega \times U$.

(2) $L: \mathbb{R}^{n} \times \mathbb{R}^{m} \rightarrow \mathbb{R}$ satisfies $\left|L\left(x^{\prime}, u^{\prime}\right)-L(x, u)\right| \leq L_{L}\left[\left\|x^{\prime}-x\right\|+\left\|u^{\prime}-u\right\|\right]$ and $1 \leq|L(x, u)| \leq M_{L}, x \in \Omega, u \in U$, for some $L_{L}, M_{L}>0$.

(3) $h: \mathbb{R}^{n} \rightarrow \mathbb{R}$ satisfies $\left|h\left(x^{\prime}\right)-h(x)\right| \leq L_{h}\left\|x^{\prime}-x\right\|$ for some $L_{h}>0$, and $h(x) \geq 0$ for all $x \in \Omega$. Let $M_{h}$ be the upper bound of $|h(x)|$ on $\Omega$.

The value function or optimal cost-to-go function $V: \mathbb{R}^{n} \rightarrow \mathbb{R}$ is given by

$$
V(y)=\inf _{\mu \in \mathcal{U}_{y}} J(y, \mu)
$$

for $y \in \Omega \backslash \Omega_{f}$, and by $V(y)=h(y)$ for $y \in \Omega_{f}$. A control $\mu$ is called $\epsilon$-optimal for $x$ if $J(x, \mu) \leq V(x)+\epsilon$. It is well-known [7] that $V$ satisfies the HamiltonJacobi-Bellman (HJB) equation

$$
-\inf _{u \in U}\left\{L(x, u)+\frac{\partial V}{\partial x} f(x, u)\right\}=0
$$

at each point of $\mathcal{R}$ at which it is differentiable. The HJB equation is an infinitesimal version of the equivalent Dynamic Programming Principle (DPP) which says that

$$
\begin{aligned}
& V(x)=\inf _{\mu \in \mathcal{U}_{x}}\left\{\int_{0}^{t} L\left(\phi_{s}(x, \mu), \mu(s)\right) d s+V\left(\phi_{t}(x, \mu)\right)\right\}, \\
& V(x)=h(x) \\
& x \in \Omega_{f} .
\end{aligned}
$$

The subject of assiduous effort has been that the HJB equation may not have a $C^{1}$ solution. This gap in the theory was closed by the inception of the concept of viscosity solution [6], which can be shown to provide the unique solution of (5) without any differentiability assumption. In particular, a bounded uniformly continuous function $V$ is called a viscosity solution of HJB provided, for each $\psi \in C^{1}\left(\mathbb{R}^{n}\right)$, the following hold: 
(i) if $V-\psi$ attains a local maximum at $x_{0} \in \mathbb{R}^{n}$, then

$$
-\inf _{u \in U}\left\{L\left(x_{0}, u\right)+\frac{\partial \psi}{\partial x}\left(x_{0}\right) f\left(x_{0}, u\right)\right\} \leq 0
$$

(ii) if $V-\psi$ attains a local minimum at $x_{1} \in \mathbb{R}^{n}$, then

$$
-\inf _{u \in U}\left\{L\left(x_{1}, u\right)+\frac{\partial \psi}{\partial x}\left(x_{1}\right) f\left(x_{1}, u\right)\right\} \geq 0 .
$$

Assumption 2.2. For every $\epsilon>0$ and $x \in \mathcal{R}$, there exists $N_{\epsilon}>0$ and an admissible piecewise constant $\epsilon$-optimal control $\mu$ having at most $N_{\epsilon}$ discontinuities and such that $\phi_{t}(x, \mu)$ is transverse to $\partial \Omega_{f}$.

The transversality assumption implies that the viscosity solution is continuous at the boundary of the target set, a result needed in proving uniform continuity of $V$. The finite switching assumption holds under mild assumptions such as Lipschitz continuity of the vector field and cost functions, and is based on approximating measurable functions by piecewise constant functions.

\section{Hybrid system}

The approach we propose for solving the continuous optimal control problem first requires a mapping to a hybrid system and, second, employs a bisimulation of the hybrid system to formulate a dynamic programming problem on the quotient system. In this section we define the hybrid optimal control problem. First, we discretize $U$ by defining a finite set $\Sigma_{\delta} \subset U$ which has a mesh size

$$
\delta:=\sup _{u \in U} \min _{\sigma \in \Sigma_{\delta}}\|u-\sigma\| .
$$

We define the hybrid automaton $H:=\left(\Sigma \times \mathbb{R}^{n}, \Sigma, D, E_{h}, G, R\right)$ with the following components.

State set $\Sigma \times \mathbb{R}^{n}$ consists of the finite set $\Sigma=\Sigma_{\delta} \cup\left\{\sigma_{f}\right\}$ of control locations and $n$ continuous variables $x \in \mathbb{R}^{n}$. $\sigma_{f}$ is a terminal location when the continuous dynamics are stopped (in the same sense that the dynamics are "stopped" in the continuous optimal control problem).

Events $\Sigma=\Sigma_{\delta} \cup\left\{\sigma_{f}\right\}$ is a finite set of control event labels.

Vector fields $D: \Sigma \rightarrow \mathcal{X}\left(\mathbb{R}^{n}\right)$ is a function assigning an autonomous vector field to each location. We use the notation $D(\sigma)=f_{\sigma}$.

Control switches $E_{h} \subset \Sigma \times \Sigma$ is a set of control switches. $e=\left(\sigma, \sigma^{\prime}\right)$ is a directed edge between a source location $\sigma$ and a target location $\sigma^{\prime}$. If $E_{h}(\sigma)$ denotes the set of edges that can be enabled at $\sigma \in \Sigma$, then $E_{h}(\sigma):=$ $\left\{\left(\sigma, \sigma^{\prime}\right) \mid \sigma^{\prime} \in \Sigma \backslash \sigma\right\}$ for $\sigma \in \Sigma_{\delta}$ and $E_{h}\left(\sigma_{f}\right)=\emptyset$. Thus, from a source location not equal to $\sigma_{f}$, there is an edge to every other location (but not itself), while location $\sigma_{f}$ has no outgoing edges.

Enabling conditions $G: E_{h} \rightarrow\left\{g_{e}\right\}_{e \in E_{h}}$ is a function assigning to each edge an enabling (or guard) condition $g \subset \mathbb{R}^{n}$. We use the notation $G(e)=g_{e}$. 
Reset conditions $R: E_{h} \rightarrow\left\{r_{e}\right\}_{e \in E_{h}}$ is a function assigning to each edge a reset condition, $r_{e}: \mathbb{R}^{n} \rightarrow 2^{\mathbb{R}^{n}}$, where we use the notation $R(e)=r_{e}$.

Semantics. A state is a pair $(\sigma, x), \sigma \in \Sigma$ and $x \in \mathbb{R}^{n}$. In location $\sigma \in \Sigma_{\delta}$ the continuous state evolves according to the vector field $f(x, \sigma)$. In location $\sigma_{f}$, the vector field is $\dot{x}=f\left(x, \mu_{f}\right)$ where $\mu_{f}$ is the (not necessarily constant) control of the terminal location. Trajectories of $H$ evolve in steps of two types. A $\sigma$-step is a binary relation $\stackrel{\sigma}{\rightarrow} \subset\left(\Sigma \times \mathbb{R}^{n}\right) \times\left(\Sigma \times \mathbb{R}^{n}\right)$, and we write $(\sigma, x) \stackrel{\sigma^{\prime}}{\rightarrow}\left(\sigma^{\prime}, x^{\prime}\right)$ iff (1) $e=\left(\sigma, \sigma^{\prime}\right) \in E_{h},(2) x \in g_{e}$, and $(3) x^{\prime}=r_{e}(x)$. The transition $(\sigma, x) \stackrel{\sigma^{\prime}}{\rightarrow}\left(\sigma^{\prime}, x^{\prime}\right)$ is taken at the first time in location $\sigma$ when the control event label is $\sigma^{\prime}$ and $x \in g_{e}$ for $e=\left(\sigma, \sigma^{\prime}\right)$. A $t$-step is a binary relation $\stackrel{t}{\rightarrow} \subset\left(\Sigma \times \mathbb{R}^{n}\right) \times\left(\Sigma \times \mathbb{R}^{n}\right)$, and we write $(\sigma, x) \stackrel{t}{\rightarrow}\left(\sigma^{\prime}, x^{\prime}\right)$ iff $(1) \sigma=\sigma^{\prime},(2)$ at $t=0, x^{\prime}=x$, and (3) for $t \geq 0, x^{\prime}=\phi_{t}(x, \sigma)$, where $\phi_{t}(x)=f\left(\phi_{t}(x, \sigma), \sigma\right)$. A hybrid control is a finite or infinite sequence of labels $\omega=\omega_{1} \omega_{2} \ldots$, with $\omega_{i} \in \Sigma \cup \mathbb{R}^{+} . \omega_{i} \in \mathbb{R}^{+}$is the duration of the $t$-step at step $i$. The set of hybrid controls is denoted $\mathcal{S}$. A hybrid trajectory $\pi$ over $\omega \in \mathcal{S}$ is a finite or infinite sequence $\pi:\left(\sigma_{0}, x_{0}\right) \stackrel{\omega_{1}}{\rightarrow}\left(\sigma_{1}, x_{1}\right) \stackrel{\omega_{2}}{\longrightarrow}$ $\left(\sigma_{2}, x_{2}\right) \stackrel{\omega_{3}}{\rightarrow} \ldots$ where $\left(\sigma_{i}, x_{i}\right) \in \Sigma \times \mathbb{R}^{n}$. Trajectory $\pi$ is accepted by $H$ iff $\forall i$, $\left(\sigma_{i}, x_{i}\right) \stackrel{\omega_{i+1}}{\longrightarrow}\left(\sigma_{i+1}, x_{i+1}\right)$ is either a $t$-step or $\sigma$-step of $H$. Let $\pi$ be the trajectory (not necessarily accepted by $H$ ) starting at $(\sigma, x) \in \Sigma \times \Omega$ and defined over $\omega \in \mathcal{S}$. We say $\omega$ is admissible for $(\sigma, x)$ on interval $[0, T]$ if (1) $\pi$ remains in $\Sigma \times \Omega$ for $t \in[0, T]$, and (2) corresponding to $\omega$ is a piecewise constant control $\mu_{\omega}(t)$ (with a finite number of discontinuities in finite time). Let $\mathcal{S}_{(\sigma, x)}$ be the set of admissible controls for $(\sigma, x)$.

\subsection{Hybrid optimal synthesis}

We want to synthesize enabling conditions so that for each $y \in \mathcal{R}$, the cost-togo from $y$ well-approximates the viscosity solution at $y$ of HJB. This requires posing a hybrid optimal synthesis problem. We define a hybrid cost-to-go function $J_{H}: \Sigma \times \mathbb{R}^{n} \times \mathcal{S} \rightarrow \mathbb{R}$ as follows. For $\omega \in \mathcal{S}_{(\sigma, x)}$,

$$
J_{H}((\sigma, x), \omega)=J\left(x, \mu_{\omega}\right) .
$$

The hybrid value function $V_{H}: \Sigma \times \mathbb{R}^{n} \rightarrow \mathbb{R}$ is

$$
V_{H}((\sigma, x))=\inf _{\omega \in \mathcal{S}_{(\sigma, x)}} J_{H}((\sigma, x), \omega) .
$$

\section{Hybrid optimal synthesis problem:}

Given $H$ and $0<\epsilon^{1}<\epsilon^{2}$, synthesize $g_{e}, e \in E_{h}$, subject to:

1. $g_{e}=\Omega_{f}$ if $e=\left(\sigma, \sigma_{f}\right), \sigma \in \Sigma_{\delta}$.

2. For each $e \in E_{h}, g_{e} \subseteq \Omega$.

3. For all $\omega \in \mathcal{S}$ and $(\sigma, x) \in \Sigma \times \Omega$ such that $V_{H}((\sigma, x))<\infty, \pi_{(\sigma, x)}$ is accepted by $H$ if $\omega$ is admissible and $\epsilon^{1}$-optimal for $(\sigma, x)$.

4. For all $\omega \in \mathcal{S}$ and $(\sigma, x) \in \Sigma \times \Omega, \pi_{(\sigma, x)}$ is not accepted by $H$ if either $\omega$ is not admissible for $(\sigma, x), \omega$ is not $\epsilon^{2}$-optimal for $(\sigma, x)$, or $V_{H}((\sigma, x))=\infty$. 


\section{Construction of bisimulation}

We propose to solve the hybrid optimal control problem using the bisimulation of $H$. In this section we define bisimulation and the quotient system that is obtained from it.

Let $\lambda$ represent a $t$-step corresponding to some $t \in \mathbb{R}^{+}$. A bisimulation of $H$ is an equivalence relation $\simeq \subset\left(\Sigma_{\delta} \times \mathbb{R}^{n}\right) \times\left(\Sigma_{\delta} \times \mathbb{R}^{n}\right)$ such that for all states $p_{1}, p_{2} \in \Sigma_{\delta} \times \mathbb{R}^{n}$, if $p_{1} \simeq p_{2}$ and $\sigma \in \Sigma_{\delta} \cup\{\lambda\}$, then if $p_{1} \stackrel{\sigma}{\rightarrow} p_{1}^{\prime}$, there exists $p_{2}^{\prime}$ such that $p_{2} \stackrel{\sigma}{\rightarrow} p_{2}^{\prime}$ and $p_{1}^{\prime} \simeq p_{2}^{\prime}$. If $\simeq$ is finite, the quotient system is a finite automaton.

Since the dynamics are restricted to the set $\Omega$, the set of interesting equivalence classes of $\simeq$, denoted $Q$, are those that intersect $\Sigma_{\delta} \times \operatorname{cl}(\Omega)$. For each $q \in Q$ we define a distinguished point $(\sigma, \xi) \in q$. We associate $q$ with its distinguished point by the notation $q=[(\sigma, \xi)]$. It is now possible to define the enabling and reset conditions of $H$ in terms of $Q$. In particular, the enabling conditions of $H$ are synthesized as subsets of $Q$ while the reset conditions are defined as follows. For $e=\left(\sigma, \sigma^{\prime}\right)$

$$
r_{e}(x)=\left\{y \mid \exists \xi \cdot[(\sigma, x)]=[(\sigma, \xi)] \wedge\left[\left(\sigma^{\prime}, \xi\right)\right]=\left[\left(\sigma^{\prime}, y\right)\right]\right\}
$$

That is, $r_{e}(x)$ is the projection to $\mathbb{R}^{n}$ of the set of equivalence classes $\left[\left(\sigma^{\prime}, y\right)\right]$ such that the projection to $\mathbb{R}^{n}$ of $\left[\left(\sigma^{\prime}, y\right)\right]$ and $[(\sigma, x)]$ have nonempty intersection. This definition in effect gives an over-approximation of the identity map in terms of the equivalence classes of $\simeq$ and will introduce non-determinacy in the finite automaton. Notice also that (6) encodes information about the bisimulation in $H$. This sequence of steps is not typical; it is characteristic of our synthesis procedure. We define a mesh size on $Q$ by $\delta_{Q}=\max _{q \in Q} \sup _{(\sigma, x),(\sigma, y) \in q}\{\|x-y\|\}$. Finally, for each $q=[(\sigma, \xi)] \in Q$ we associate the duration $\tau_{q}$, the maximum time to traverse $q$ using constant control $\sigma$. That is, $\tau_{q}=\sup _{(\sigma, x),(\sigma, y) \in q}\{t \mid y=$ $\left.\phi_{t}(x, \sigma)\right\}$.

Geometric construction. We give a brief review of the method developed in [4] for obtaining bisimulations. We require the following (related) assumptions on the vector fields on $\operatorname{cl}(\Omega)$.

\section{Assumption 4.1.}

(1) $n-1$ first integrals can be defined analytically on $\Omega$ for each $f(x, \sigma)$, $\sigma \in \Sigma_{\delta}$

(2) There exists $m_{f}>0$ such that $\|f(x, u)\| \geq m_{f}$ for all $x \in \operatorname{cl}(\Omega), u \in U$.

A bisimulation of $\Sigma_{\delta} \times \mathbb{R}^{n}$ is constructed using a set of simple, co-dimension one tangential foliations with associated submersions $\gamma_{i}^{\sigma}(x)=y_{i}^{\sigma}, i=1, \ldots, n-1$ and a simple co-dimension one transversal foliation with submersion $\gamma_{n}^{\sigma}=y_{n}^{\sigma}$, such that $\left(y_{1}^{\sigma}, \ldots, y_{n}^{\sigma}\right)$ form a set of euclidean coordinates for each $\sigma \in \Sigma_{\delta}$. We discretize the foliations by selecting a finite set of leaves. Fix $k \in Z^{+}$and let $\Delta=\frac{1}{2^{k}}$. Define

$$
C_{k}=\{0, \pm \Delta, \pm 2 \Delta, \ldots, \pm 1\}
$$


Each $y_{i}^{\sigma}=c$ for $c \in C_{k}, i=1, \ldots, n$ defines a hyperplane denoted $\tilde{W}_{i, c}^{\sigma}$, and a submanifold $W_{i, c}^{\sigma}=\left(\gamma^{\sigma}\right)^{-1}\left(\tilde{W}_{i, c}^{\sigma}\right)$. The collection of submanifolds for $\sigma \in \Sigma_{\delta}$ is

$$
\mathcal{W}_{k}^{\sigma}=\left\{W_{i, c}^{\sigma} \mid c \in C_{k}, i \in\{1, \ldots, n\}\right\} .
$$

$\Omega \backslash \mathcal{W}_{k}^{\sigma}$ is the union of $2^{n(k+1)}$ disjoint open sets $\mathcal{V}_{k}^{\sigma}=\left\{V_{j}^{\sigma}\right\}$. We define the equivalence relation $\simeq$ on $\Sigma_{\delta} \times \mathbb{R}^{n}$ as follows: $(\sigma, x) \simeq\left(\sigma^{\prime}, x^{\prime}\right)$ iff $(1) \sigma=\sigma^{\prime}$ and (2) $x \in W$ iff $x^{\prime} \in W$, and $x \in V$ iff $x^{\prime} \in V$, for all $W \in \mathcal{W}_{k}^{\sigma}$ and $V \in \mathcal{V}_{k}^{\sigma}$.

\section{Discrete problem}

In this section we transform the hybrid optimal control problem to a dynamic programming problem on a non-deterministic finite automaton, for which an algorithmic solution may be found. Consider the class of non-deterministic automata with cost structure represented by the tuple $A=\left(Q, \Sigma_{\delta}, E, o b s, Q_{f}, \hat{L}, \hat{h}\right)$. $Q$ is the state set, as above, and $\Sigma_{\delta}$ is the set of control labels as before. obs : $E \rightarrow \Sigma_{\delta}$ is a map that assigns a control label to each edge and is given by $\operatorname{obs}(e)=\sigma^{\prime}$, where $e=\left(q, q^{\prime}\right), q=[(\sigma, \xi)]$ and $q^{\prime}=\left[\left(\sigma^{\prime}, \xi^{\prime}\right)\right] . Q_{f}$ is the target set given by the over-approximation of $\Omega_{f}, Q_{f}=\left\{q \in Q \mid \exists x \in \Omega_{f} .(\sigma, x) \in q\right\}$.

$E \subseteq Q \times Q$ is the transition relation encoding $t$-steps and $\sigma$-steps of $H$. $A$ will be used to synthesize $g_{e}$ of $H$, so $E$ includes all possible edges between locations. The synthesis procedure on $A$ will involve trimming undesirable edges. Thus, $\left(q, q^{\prime}\right) \in E$, where $q, q^{\prime} \in Q, q=[(\sigma, \xi)]$ and $q^{\prime}=\left[\left(\sigma^{\prime}, \xi^{\prime}\right)\right]$ if either (a) $\sigma=\sigma^{\prime}$, there exists $x \in \Omega$ such that $(\sigma, x) \in Q$, and there exists $\tau>0$ such that $\forall t \in[0, \tau],\left(\sigma, \phi_{t}(x, \sigma)\right) \in q$ and $\left(\sigma, \phi_{\tau+\epsilon}(x, \sigma)\right) \in q^{\prime}$ for arbitrarily small $\epsilon>0$, or (b) $\sigma=\sigma^{\prime}$, there exists $x \in \Omega$ such that $(\sigma, x) \in Q$, and there exists $\tau>0$ such that $\forall t \in[0, \tau),\left(\sigma, \phi_{t}(x, \sigma)\right) \in q$ and $\left(\sigma, \phi_{\tau}(x, \sigma)\right) \in q^{\prime}$, or (c) $\sigma \neq \sigma^{\prime}$ and there exists $x \in \Omega$ such that $(\sigma, x) \in Q$ and $\left(\sigma^{\prime}, x\right) \in q^{\prime}$. Cases (a) and (b) say that from a point in $q, q^{\prime}$ is the first state (different from $q$ ) reached after following the flow of $f(x, \sigma)$ for some time. Case (c) says that an edge exists between $q$ and $q^{\prime}$ if their projections to $\mathbb{R}^{n}$ have non-empty intersection.

Let $e=\left(q, q^{\prime}\right)$ with $q=[(\sigma, \xi)]$ and $q^{\prime}=\left[\left(\sigma^{\prime}, \xi^{\prime}\right)\right] . \hat{L}: E \rightarrow \mathbb{R}$ is the discrete instantaneous cost given by

$$
\hat{L}(e):= \begin{cases}\tau_{q} L(\xi, \sigma) & \text { if } \quad \sigma=\sigma^{\prime} \\ 0 & \text { if } \sigma \neq \sigma^{\prime}\end{cases}
$$

This definition reflects that no cost is incurred for control switches. $\hat{h}: Q \rightarrow \mathbb{R}$ is the discrete terminal cost given by

$$
\hat{h}(q):=h(\xi) .
$$

The domain of $\hat{h}$ can be extended to $\Omega$, with a slight abuse of notation, by $\hat{h}(x):=\hat{h}(q)$ where $q=\arg \min _{q^{\prime}}\left\{\left\|x-\xi^{\prime}\right\| \mid q^{\prime}=\left[\left(\sigma^{\prime}, \xi^{\prime}\right)\right]\right\}$. 


\subsection{Semantics}

A transition or step of $A$ from $q=[(\sigma, \xi)] \in Q$ to $q^{\prime}=\left[\left(\sigma^{\prime}, \xi^{\prime}\right)\right] \in Q$ with observation $\sigma^{\prime} \in \Sigma_{\delta}$ is denoted $q \stackrel{\sigma^{\prime}}{\rightarrow} q^{\prime}$. If $\sigma \neq \sigma^{\prime}$ the transition is referred to as a control switch; otherwise, it is referred to as a time step. If $E(q)$ is the set of edges that can be enabled from $q \in Q$, then for $\sigma \in \Sigma_{\delta}$,

$$
E_{\sigma}(q)=\{e \in E(q) \mid o b s(e)=\sigma\} .
$$

If $\left|E_{\sigma}(q)\right|>1$, then we say that $e \in E_{\sigma}(q)$ is unobservable in the sense that when control event $\sigma$ is issued, it is unknown which edge among $E_{\sigma}(q)$ is taken. If $\sigma=\sigma^{\prime}$, then $\left|E_{\sigma}(q)\right|=1$, by the uniqueness of solutions of ODE's and by the definition of bisimulation.

A control policy $c: Q \rightarrow \Sigma_{\delta}$ is a map assigning a control event to each state; $c(q)=\sigma$ is the control event issued when the state is at $q$. A trajectory $\pi$ of $A$ over $c$ is a sequence $\pi=q_{0} \stackrel{\sigma_{1}}{\rightarrow} q_{1} \stackrel{\sigma_{2}}{\rightarrow} q_{2} \stackrel{\sigma_{3}}{\rightarrow} \ldots, q_{i} \in Q$. A trajectory is non-Zeno if between any two non-zero duration time steps there are a finite number of control switches and zero duration time steps. Let $\Pi_{c}(q)$ be the set of trajectories starting at $q$ and applying control policy $c$, and let $\Pi_{c}(q)$ be the set of trajectories starting at $q$, applying control policy $c$, and eventually reaching $Q_{f}$. If for every $q \in Q, \pi \in \Pi_{c}(q)$ is non-Zeno then we say $c$ is an admissible control policy. The set of all admissible control policies for $A$ is denoted $\mathcal{C}$.

A control policy $c$ is said to have a loop if $A$ has a trajectory $q_{0} \stackrel{c\left(q_{0}\right)}{\longrightarrow} q_{1} \stackrel{c\left(q_{1}\right)}{\longrightarrow}$ $\ldots \stackrel{c\left(q_{m-1}\right)}{\rightarrow} q_{m}=q_{0}, q_{i} \in Q$. A control policy has a Zeno loop if it has a loop made up of control switches and/or zero duration time steps only. One can show that a control policy is admissible iff it has no Zeno loops.

\subsection{Dynamic programming}

In this section we formulate the dynamic programming problem on $A$. This involves defining a cost-to-go function and a value function that minimizes it over control policies suitable for non-deterministic automata.

Suppose $\pi=q_{0} \stackrel{\sigma_{1}}{\rightarrow} q_{1} \rightarrow \ldots \rightarrow q_{N-1} \stackrel{\sigma_{N}}{\rightarrow} q_{N} \in \Pi$, where $q_{i}=\left[\left(\sigma_{i}, \xi_{i}\right)\right]$ and $\pi$ takes the sequence of edges $e_{1} e_{2} \ldots e_{N}$. We define a discrete cost-to-go $\hat{J}: Q \times \mathcal{C} \rightarrow \mathbb{R}$ by

$$
\hat{J}(q, c)= \begin{cases}\max _{\pi \in \tilde{\Pi}_{c}(q)}\left\{\sum_{j=1}^{N_{\pi}} \hat{L}\left(e_{j}\right)+\hat{h}\left(q_{N_{\pi}}\right)\right\} & \text { if } \Pi_{c}(q)=\tilde{\Pi}_{c}(q) \\ \infty & \text { otherwise }\end{cases}
$$

where $N_{\pi}=\min \left\{j \geq 0 \mid q_{j} \in Q_{f}\right\}$. We take the maximum over $\tilde{\Pi}_{c}(q)$ because of the non-determinacy of $A$ : it is uncertain which among the (multiple) trajectories allowed by $c$ will be taken so we must assume the worst-case situation. The discrete value function $\hat{V}: Q \rightarrow \mathbb{R}$ is

$$
\hat{V}(q)=\min _{c \in \mathcal{C}} \hat{J}(q, c)
$$


for $q \in Q \backslash Q_{f}$ and $\hat{V}(q)=\hat{h}(q)$ for $q \in Q_{f}$. We show in Proposition 1 that $\hat{V}$ satisfies a DPP that takes into account the non-determinacy of $A$ and ensures that optimal control policies are admissible. This DPP describes the accumulation of cost over one step to be the worst case cost among edges that have the same label. Let $\mathcal{A}_{q}$ be the set of control assignments $c(q) \in \Sigma_{\delta}$ at $q$ such that $c$ is admissible.

Proposition 1. $\hat{V}$ satisfies

$$
\begin{aligned}
& \hat{V}(q)=\min _{c(q) \in \mathcal{A}_{q}}\left\{\max _{e=\left(q, q^{\prime}\right) \in E_{c(q)}(q)}\left\{\hat{L}(e)+\hat{V}\left(q^{\prime}\right)\right\}\right\}, \quad q \in Q \backslash Q_{f} \\
& \hat{V}(q)=\hat{h}(q), \quad q \in Q_{f} \text {. }
\end{aligned}
$$

\subsection{Synthesis of $g_{e}$}

The synthesis of enabling conditions or controller synthesis is typically a postprocessing step of a backward reachability analysis (see, for example, [12]). This situation prevails here as well: equations (10)-(11) describe a backward analysis to construct an optimal policy $c \in \mathcal{C}$. Once $c$ is known the enabling conditions of $H$ are extracted as follows.

Consider each $e=\left(\sigma, \sigma^{\prime}\right) \in E$ of $H$ with $\sigma \neq \sigma^{\prime}$. There are two cases. If $\sigma^{\prime} \neq \sigma_{f}$ then $g_{e}=\left\{x \mid(\sigma, x) \in q, q \in Q \wedge c(q)=\sigma^{\prime}\right\}$. That is, if the control policy designates switching from $q \in Q$ with label $\sigma$ to $q^{\prime} \in Q$ with label $\sigma^{\prime}$, then the corresponding enabling condition in $H$ includes the projection to $\mathbb{R}^{n}$ of $q$. The second case when $\sigma^{\prime}=\sigma_{f}$ is for edges going to the terminal location of $H$. Then $g_{e}=\left\{x \mid(\sigma, x) \in q, q \in Q_{f}\right\}$.

\section{Main Result}

We will prove that $\hat{V}$ converges to $V$, the viscosity solution of the HJB equation, as $\delta_{Q}, \delta \rightarrow 0$. The proof will be carried out in three steps. In the first step we consider restricting the set of controls to piecewise constant functions, whose constant intervals are a function of the state. In the second step we introduce the discrete approximations of $L$ and $h$. In the last step we introduce the discrete states $Q$ and consider the non-determinacy of $A$.

In the sequel we make use of a filtration of control sets $\Sigma_{k} \equiv \Sigma_{\delta_{k}}$ corresponding to a sequence $\delta_{k} \rightarrow 0$ as $k \rightarrow \infty$, in such a manner that $\Sigma_{k} \subset \Sigma_{k+1}$. Considering (8), we define a filtration of families of submanifolds such that $\mathcal{W}_{k}^{\sigma} \subset \mathcal{W}_{k+1}^{\sigma}$, for each $\sigma \in \Sigma_{k}$.

\section{Step 1: piecewise constant controls.}

In the first step we define a class of piecewise constant functions that depend on the state and show that the value function which minimizes the cost-to-go over this class converges to the viscosity solution of HJB as $\delta_{k} \rightarrow 0$. The techniques of this step are based on those in Bardi and Capuzzo-Dolcetta [1] and are related to those in [5]. 
We consider the optimal control problem (2)-(4) when the set of admissible controls is $\mathcal{U}_{k}^{1}$, piecewise constant functions consisting of finite sequences of control labels $\sigma \in \Sigma_{k}$ and each $\sigma$ is applied for a time $\tau(\sigma, x)$. Let $(\sigma, x) \in q$ for some $q \in Q$ and define $\tau(\sigma, x)$ to be the minimum of the time it takes the trajectory starting at $x$ and using control $\sigma \in \Sigma_{k}$ to reach (ta) $\partial \Omega_{f}$, and (tb) some $x^{\prime}$ such that $\left(\sigma, x^{\prime}\right) \notin q$. If a trajectory is at $x_{i}$ at the start of the $(i+1)$ th step, then the control $\sigma_{i+1}$ is applied for time $\tau_{i+1}:=\tau\left(\sigma_{i+1}, x_{i}\right)$ and $x_{i+1}=\phi_{\tau_{i+1}}\left(x_{i}, \sigma_{i+1}\right)$.

Let

$$
\mathcal{R}_{k}^{1}:=\left\{x \in \mathbb{R}^{n} \mid \exists \mu \in \mathcal{U}_{k}^{1} . T(x, \mu)<\infty\right\} .
$$

We define the cost-to-go function $J_{k}^{1}: \Omega \times \mathcal{U}_{k}^{1} \rightarrow \mathbb{R}$ as follows. For $x \in \Omega$ and $\mu=\sigma_{1} \sigma_{2} \ldots \in \mathcal{U}_{k}^{1}$, if $T(x, \mu)<\infty$ then

$$
J_{k}^{1}(x, \mu)=\sum_{j=1}^{N} \int_{0}^{\tau\left(\sigma_{j}, x_{j-1}\right)} L\left(\phi_{s}\left(x_{j-1}, \sigma_{j}\right), \sigma_{j}\right) d s+h\left(x_{N}\right)
$$

where $N=\min \left\{j \geq 0 \mid x_{j} \in \partial \Omega_{f}\right\} . J_{k}^{1}(x, \mu)=\infty$, otherwise. We define the value function $V_{k}^{1}: \mathbb{R}^{n} \rightarrow \mathbb{R}$ as follows. For $x \in \Omega \backslash \Omega_{f}$,

$$
V_{k}^{1}(x)=\inf _{\mu \in \mathcal{U}_{k}^{1}} J_{k}^{1}(x, \mu)
$$

and for $x \in \Omega_{f}, V_{k}^{1}(x)=h(x)$.

$\left\{V_{k}^{1}\right\}$ forms a family of equibounded, locally equicontinuous functions. It can then be shown that, along some subsequence $k_{n}, V_{k_{n}}^{1}$ converges to a continuous function $V_{*}$. Moreover, the following holds:

Proposition 2. $V_{*}$ is the unique viscosity solution of HJB.

\section{Step 2: approximate cost functions.}

In this step we keep the semantics on piecewise constant controls of Step 1 but replace cost functions $L$ and $h$ by approximations $L^{2}$ and $\hat{h}$. We define the cost-to-go function $J_{k}^{2}: \Omega \times \mathcal{U}_{k}^{1} \rightarrow \mathbb{R}$ as follows. First, we define an approximate instantaneous cost $L^{2}: \Omega \times \Sigma_{k} \rightarrow \mathbb{R}$ given by

$$
L^{2}(x, \sigma):=\hat{L}(q)
$$

where $(\sigma, x) \in q$. For $x \in \Omega$ and $\mu=\sigma_{1} \sigma_{2} \ldots \in \mathcal{U}_{k}^{1}$, if $T(x, \mu)<\infty$ then

$$
J_{k}^{2}(x, \mu)=\sum_{j=1}^{N} L^{2}\left(x_{j-1}, \sigma_{j}\right)+\hat{h}\left(x_{N}\right)
$$

where $N=\min \left\{j \geq 0 \mid x_{j} \in \partial \Omega_{f}\right\}$. We define a value function $V_{k}^{2}: \mathbb{R}^{n} \rightarrow \mathbb{R}$ as follows. For $x \in \Omega \backslash \Omega_{f}$,

$$
V_{k}^{2}(x)=\inf _{\mu \in \mathcal{U}_{k}^{1}} J_{k}^{2}(x, \mu)
$$

and for $x \in \Omega_{f}, V_{k}^{2}(x)=\hat{h}(x)$. For $x \in \Omega$ such that $V_{k}^{2}(x)<\infty, V_{k}^{2}$ satisfies the DPP $V_{k}^{2}(x)=\min _{\sigma \in \Sigma_{k}}\left\{L^{2}(x, \sigma)+V_{k}^{2}\left(\phi_{\tau(\sigma, x)}(x, \sigma)\right)\right\}$. 


\section{Remark 6.1.}

For each $x \in \cup_{k} \mathcal{R}_{k}^{1}$ and $\epsilon>0$ there exists $m \in \mathbb{Z}^{+}$and $\mu \in \mathcal{U}_{m}^{1}$ such that $\mu$ is an $\epsilon$-optimal control for $x$ w.r.t. $V^{1}$ satisfying Assumptions 2.2. This follows from Assumptions 2.2, $V_{k}^{1}(x) \geq V(x)$, and the fact that we can well-approximate an $\epsilon$-optimal control for $V$ by a control in $\mathcal{U}_{m}^{1}$, for large enough $m$.

Proposition 3. Let $k_{0} \in \mathbb{Z}^{+}, x \in \mathcal{R}_{k_{0}}^{1}$, and $\mu \in \mathcal{U}_{k_{0}}^{1}$ be an $\epsilon$-optimal control for $x$. Then $\left|J_{k}^{1}(x, \mu)-J_{k}^{2}(x, \mu)\right| \rightarrow 0$ as $k \rightarrow \infty$.

Proof. First, we require two facts which are stated without proof, for brevity.

Fact 1. If $\delta_{k}<\frac{m_{f}}{L_{f}}$, then for all $q \in Q$,

$$
\tau_{q} \leq \frac{\delta_{k}}{m_{f}-L_{f} \delta_{k}} .
$$

For the next fact, we require a definition. let $C_{k}$ be as in (7) and $\gamma_{n}^{\sigma}$ the transversal foliation of $\dot{x}=f(x, \sigma)$. For $\sigma \in \Sigma_{k}$, define the region in $\mathbb{R}^{n}$

$$
M_{c}^{\sigma}:=\left\{x \in\left(\gamma_{n}^{\sigma}\right)^{-1}(c) \mid c \in C_{k}\right\} .
$$

Fact 2. Let $x, x^{\prime} \in M_{c}^{\sigma}$ for some $c \in C_{k}$ and $\sigma \in \Sigma_{k}$. Let $\tau, \tau^{\prime}$ be times such that $\phi_{\tau}(x, \sigma), \phi_{\tau^{\prime}}\left(x^{\prime}, \sigma\right) \in M_{c+\Delta}^{\sigma}$. Then $\left|\tau-\tau^{\prime}\right| \leq c_{\gamma} \tau \delta_{k}$ for some $c_{\gamma}>0$.

Now we have

$$
\begin{aligned}
\left|J_{k}^{1}(x, \mu)-J_{k}^{2}(x, \mu)\right| \leq & \mid \sum_{j=1}^{N}\left[\int_{0}^{\tau\left(\sigma_{j}, x_{j-1}\right)} L\left(\phi_{s}\left(x_{j-1}, \sigma_{j}\right), \sigma_{j}\right) d s\right]+h\left(x_{N}\right) \\
& -\sum_{j=1}^{N}\left[\tau_{q_{j-1}} L\left(\xi_{j-1}, \sigma_{j}\right)\right]-\hat{h}\left(x_{N}\right) \mid
\end{aligned}
$$

where $\left(x_{j-1}, \sigma_{j}\right) \in q_{j-1}$ and $q_{j-1}=\left[\left(\xi_{j-1}, \sigma_{j}\right)\right]$. There exists $\xi_{N}$ such that $\hat{h}\left(x_{N}\right)=h\left(\xi_{N}\right)$ and $\left\|x_{N}-\xi_{N}\right\| \leq \delta_{k}$. Also, using the Mean Value Theorem, there exists $\tilde{t}$ with $\tilde{x}=\phi_{\tilde{t}}\left(x_{j-1}, \sigma_{j}\right)$ and $\left\|\tilde{x}-\xi_{j-1}\right\| \leq \delta_{k}$ such that

$$
\begin{aligned}
\left|J_{k}^{1}(x, \mu)-J_{k}^{2}(x, \mu)\right| & \leq \sum_{j=1}^{N}\left|\tau\left(\sigma_{j}, x_{j-1}\right) L\left(\tilde{x}, \sigma_{j}\right)-\tau_{q_{j-1}} L\left(\xi_{j-1}, \sigma_{j}\right)\right|+\left|h\left(x_{N}\right)-\hat{h}\left(x_{N}\right)\right| \\
& \leq \sum_{j=1}^{N} \tau_{q_{j-1}} L_{L} \delta_{k}+\sum_{j=1}^{N}\left[\tau_{q_{j-1}}-\tau\left(\sigma_{j}, x_{j-1}\right)\right] L\left(\tilde{x}, \sigma_{j}\right)+L_{h} \delta_{k} .
\end{aligned}
$$

Using Fact 1 the first term on the r.h.s. decreases linearly as $\delta_{k}$. Call the second term on the r.h.s. "B". Splitting B into sums over control switches and time steps, we have

$$
\begin{aligned}
B & \leq M_{L} \sum_{j=2}^{N}\left[\tau_{q_{j-1}}-\tau\left(\sigma_{j}, x_{j-1}\right)\right] \mathbf{1}\left(\sigma_{j}=\sigma_{j-1}\right)+M_{L} \sum_{j=1}^{N}\left[\tau_{q_{j-1}}-\tau\left(\sigma_{j}, x_{j-1}\right)\right] \mathbf{1}\left(\sigma_{j} \neq \sigma_{j-1}\right) \\
& \leq M_{L} \sum_{j=2}^{N} c_{j-1} \tau_{q_{j-1}} \delta_{k}+M_{L} \sum_{j=1}^{N} \tau_{q_{j-1}} \mathbf{1}\left(\sigma_{j} \neq \sigma_{j-1}\right)
\end{aligned}
$$


for some $c_{j-1} \in \mathbb{R}$. In the second line we used Fact 2 and the fact that $\tau_{q_{j-1}} \geq$ $\tau\left(\sigma_{j}, x_{j-1}\right)$. Using Fact 1 the first term on the r.h.s. decreases linearly as $\delta_{k}$. The second term on the r.h.s. goes to zero since $\mu$ has a fixed number of control switches for all $k \geq k_{0}$.

Step 3: discrete states and non-determinacy.

We define $\hat{V}_{k}(x):=\min _{\sigma \in \Sigma_{k}}\left\{\hat{V}_{k}(q) \mid(\sigma, x) \in q\right\}$. Also let $\hat{\mathcal{R}}_{k}=\{x \in$ $\left.\Omega \mid \hat{V}_{k}(x)<\infty\right\}$ and $\hat{\mathcal{R}}=\cup_{k} \hat{\mathcal{R}}_{k}$.

Remark 6.2.

(a) By Remark 6.1 and $V_{k}^{1}(x) \leq V_{k}^{2}(x)$, for each $x \in \cup_{k} \mathcal{R}_{k}^{1}$ and $\epsilon>0$ there exists $m_{\epsilon} \in \mathbb{Z}^{+}$and $\mu \in \mathcal{U}_{m_{\epsilon}}^{1}$ such that $\mu$ is an $\epsilon$-optimal control for $x$ w.r.t. $V_{k}^{2}$ satisfying Assumptions 2.2.

(b) $\hat{\mathcal{R}} \subset \cup_{k} \mathcal{R}_{k}^{1}$, but the converse is not true, in general.

(c) If $\mu$ is an $\epsilon$-optimal control for $x$ w.r.t. $V_{k}^{2}$, then we can assume $\phi_{t}(x, \mu)$ does not self-intersect, for if it did we can find $\tilde{\mu}$, also $\epsilon$-optimal, which eliminates loops in $\phi_{t}(x, \mu)$.

(d) $\|x-y\| \rightarrow 0$ as $k \rightarrow \infty$ for all $y \in r_{e}(x)$ and all edges $e$ of $H_{k}$, the hybrid automaton defined using $\Sigma_{k}$ and $C_{k}$ given in (7).

Proposition 4. For all $x \in \hat{\mathcal{R}},\left|\hat{V}_{k}(x)-V_{k}^{2}(x)\right| \rightarrow 0$ as $k \rightarrow \infty$.

Proof. Fix $\epsilon>0$ and $x \in \hat{\mathcal{R}}$. By Remark 6.2(a) there exists $m_{\epsilon}>0$ and an $\epsilon$-optimal control $\mu \in \mathcal{U}_{m_{\epsilon}}^{1}$ for $x$. Let us denote $\mu$ as an open loop control $\mu=$ $\left(\left(\sigma_{1}, \tau_{1}\right), \ldots,\left(\sigma_{N}, \tau_{N}\right)\right)$, where $\tau_{i}$ is the time $\sigma_{i}$ is applied. If $c$ is a policy derived using $\delta_{k}$ and $C_{k}$, for $k \geq m_{\epsilon}$, then $0 \leq \hat{V}_{k}(q)-V_{k}^{2}(x) \leq \hat{J}_{k}(q, c)-J^{2}(x, \mu)+\epsilon$, where $q=\left[\left(\sigma_{1}, x\right)\right]$. If we can show there exists $\bar{k} \geq m_{\epsilon}$ such that for $k>\bar{k}$, there exists a policy $\bar{c}$ such that $\hat{J}_{k}(q, \bar{c})-J_{k}^{2}(x, \mu)<\epsilon$ and using the fact that $\left|\hat{V}_{k}(q)-\hat{V}_{k}(x)\right| \rightarrow 0$ as $k \rightarrow \infty$, then the result follows.

Consider the set $\Psi_{k}$ of (discontinuous) trajectories $\phi_{t}(x, \tilde{\mu})$ where $\tilde{\mu} \in \mathcal{U}_{k}^{1}$ is denoted $\left(\left(\sigma_{1}, \tilde{\tau}_{1}, \ldots,\left(\sigma_{N}, \tilde{\tau}_{N}\right)\right)\right.$. Also $x_{j}^{-}=\phi_{\tilde{\tau}_{j}}\left(x_{j-1}, \sigma_{j}\right)$ and $x_{j} \in r_{e}\left(x_{j}^{-}\right)$, where $e=\left(\sigma_{j}, \sigma_{j+1}\right)$ is an edge of $H_{k}$, defined in Remark 6.2(d). We can find $\bar{k}_{1} \geq m_{\epsilon}$ such that, by Remark 6.2(d) and the transversality of $\phi_{t}(x, \mu)$ with the submanifolds where it switches controls and with $\Omega_{f}$, there exists $\tilde{\mu} \in \mathcal{U}_{k}^{1}$ such that $\phi_{t}(x, \tilde{\mu}) \in \Psi_{k}$ switches controls on the same (transversal) submanifolds and reaches $\Omega_{f}$. Let $W_{k}^{2}(\phi)=\sum_{j=1}^{N} L^{2}\left(x_{j-1}, \sigma_{j}\right)+\hat{h}\left(x_{N}\right)$. We observe that for $\phi, \phi^{\prime} \in$ $\Psi_{k}$ and $\mu \in \mathcal{U}_{\bar{k}_{1}}^{1},\left|W_{k}^{2}(\phi)-W_{k}^{2}\left(\phi^{\prime}\right)\right| \rightarrow 0$ as $k \rightarrow \infty$, using Lipschitz continuity of $L$ and $h$, Remark 6.2(d), and the fact that $\mu$ is fixed for all $k>\bar{k}_{1}$. Notice that $J^{2}(x, \mu)=W_{k}^{2}\left(\phi^{2}\right)$ for some $\phi^{2} \in \Psi_{k}$. We can define the control policy $\bar{c}$ such that automaton $A$ accepts the time abstract trajectory starting at $q$ corresponding to each trajectory of $\Psi_{k}$ and with all other control assignments of $\bar{c}$ as time steps. $\bar{c}$ is admissible because otherwise some $\phi^{\prime} \in \Psi_{k}$ would have a Zeno loop. Since $\phi^{\prime}$ approaches $\phi_{t}(x, \mu)$ as $k \rightarrow \infty$, this would imply $\phi_{t}(x, \mu)$ has a loop, contradicting Remark 6.2(c). Now we observe that $\hat{J}(q, \bar{c})=\max _{\phi \in \Psi_{k}} W_{k}^{2}(\phi):=$ $W_{k}^{2}(\bar{\phi})$. Thus, $\hat{J}_{k}(q, \bar{c})-J_{k}^{2}(x, \mu) \leq\left|W_{k}^{2}(\bar{\phi})-W_{k}^{2}\left(\phi^{2}\right)\right| \rightarrow 0$ as $k \rightarrow \infty$.

Theorem 1. For all $x \in \hat{\mathcal{R}}, \hat{V}_{k}(x) \rightarrow V(x)$ as $k \rightarrow \infty$. 


\section{Conclusion}

In this paper we have developed a methodology for the synthesis of optimal controls based on hybrid systems and bisimulations. The idea is to translate an optimal control problem to a switching problem on a hybrid system whose locations describe the dynamics when the control is constant. When the vector fields for each location of the hybrid automaton have local first integrals which can be expressed analytically we are able to define a finite bisimulation using the approach of [4]. From the finite bisimulation we obtain a (time abstract) finite automaton upon which a dynamic programming problem can be formulated that can be solved efficiently.

We are presently working on three topics that will enhance considerably the significance of our work:

- The dynamic programming problem is equivalent to a shortest path problem on a non-deterministic graph. We are in the process of carrying through the implementation issues to obtain an algorithmic solution.

- Throughout the paper we have assumed that, once the bisimulation is expressed using first integrals, the corresponding finite automaton can be constructed directly. In fact, this task is not so straightforward. We are working on the automatic generation of finite automata that give time abstract behavior of vector fields.

- If it is not possible to obtain a finite bisimulation, one may still be able to construct a finite automaton that approximates the continuous and discrete behavior of the hybrid system. But this automaton will have nondeterministic behavior that results in spurious solutions, not corresponding to the true dynamics of the hybrid system. We are working on a procedure to eliminate these spurious solutions.

\section{References}

1. M. Bardi and I. Capuzzo-Dolcetta. Optimal control and viscosity solutions of Hamilton-Jacobi-Bellman equations. Birkhäuser, Boston, 1997.

2. V.G. Boltyanskii. Sufficient conditions for optimality and the justification of the dynamic programming method. SIAM Journal of Control, 4, pp. 326-361, 1966.

3. M. Branicky, V. Borkar, S. Mitter. A unified framework for hybrid control: model and optimal control theory. IEEE Trans. AC, vol. 43, no. 1, pp. 31-45, January, 1998.

4. M. Broucke. A geometric approach to bisimulation and verification of hybrid systems. In Hybrid Systems: Computation and Control, F. Vaandrager and J. van Schuppen, eds., LNCS 1569, p. 61-75, Springer-Verlag, 1999.

5. I. Capuzzo Dolcetta and L.C. Evans. Optimal switching for ordinary differential equations. SIAM J. Control and Optimization, vol. 22, no. 1, pp. 143-161, January 1984.

6. M Crandall, P. Lions. Viscosity solutions of Hamilton-Jacobi equations. Trans. Amer. Math. Soc., vol. 277, no. 1, pp. 1-42, 1983. 
7. W.H. Fleming, R.W. Rishel. Deterministic and stochastic optimal control. Springer-Verlag, New York, 1975.

8. O. Maler, A Pnueli, J. Sifakis. On the synthesis of discrete controllers for timed systems. In Proc. STACS '95, E.W. Mayr and C. Puech, eds. LNCS 900, SpringerVerlag, p. 229-242, 1995.

9. J. Raisch. Controllability and observability of simple hybrid control systems-FDLTI plants with symbolic measurements and quantized control inputs. International Conference on Control '94, IEE, vol. 1, pp. 595-600, 1994.

10. J. Stiver, P. Antsaklis, M. Lemmon. A logical DES approach to the design of hybrid control systems. Mathemtical and computer modelling. vol. 23, no. 11-1, pp. 55-76, June, 1996.

11. H.S. Witsenhausen. A class of hybrid-state continuous-time dynamic systems. IEEE Trans. AC, vol. 11, no. 2, pp. 161 - 167, April, 1966.

12. H. Wong-Toi. The synthesis of controllers for linear hybrid automata. In Proc. 36th IEEE Conference on Decision and Control, pp. 4607-4612, 1997. 